\title{
GAME-THEORETICAL MODELS OF TEAM BUILDING
}

\author{
Dmitry A. Novikov
}

Institute of Control Sciences, Moscow, Russia,novikov@ipu.ru

\begin{abstract}
Nowadays, in spite of great amount of qualitative discussions in management about the teams' role, there are no formal models of team building and functioning. The paper considers the model, based on the concept of the reflexive game, which describes and explains team building in terms of hierarchy of agents' beliefs about types of each other. The analysis allows concluding, that the stability of team is also possible under false consistent beliefs of its members. Formal model of the team building process leads to the corresponding management problem. Copyright $\odot 2005$ IFAC
\end{abstract}

Keywords: game theory, management, stability.

\section{INTRODUCTION}

During the last decades more and more attention in management, project management, social psychology, etc., is paid to the team activity of the organization's personnel. The team is understood as the collective (the community of people, who implement joint activity and possess common interests), which is able to achieve goals autonomously and coordinately under minimal control.

Two aspects in the definition of a team are essential. The first aspect is the goals' achievement; i.e. the terminal result of joint activity is the system-forming factor for the team. The second aspect - autonomy and self-coordination of activity - means that each member of the team demonstrates the behavior, which is required in the certain situation, i.e. the behavior, expected from him by the other team members.

Nowadays, in spite of the great amount of qualitative discussions in management about the teams' role, there are no formal models of team building and functioning. The paper considers the models, based on the concept of the reflexive game (Novikov and Chkhartishvili, 2003), which describe and explain team building in terms of hierarchy of agents' beliefs about types of each other.

\section{THE MODEL}

Consider the set $N=\{1,2, \ldots, n\}$ of agents. The strategy of the $i$-th agent is the choice of his action $y_{i} \geq 0$, which requires Cobb-Douglas cost $c_{i}\left(y_{i}, r_{i}\right)=r_{i} \varphi\left(y_{i} / r_{i}\right)$, where $r_{i}>0$ is a type of this agent, which determines the efficiency of his activity, $\varphi(\cdot)$ is a monotone convex function. Assume that the goal of agents joint activity is to implement the given summarized "action" $\sum_{i \in N} y_{i}=R$ with minimal cost $\sum_{i \in N} c_{i}\left(y_{i}, r_{i}\right)$. From the game-theoretical point of view one can consider agent's goal functions to be equal to negative total cost. Without loss of generality put $R=1$. If the vector of types $r=\left(r_{1}, r_{2}, \ldots, r_{n}\right)$ is common knowledge (Myerson, 1995), then, solving the optimization problem of cost minimization, each agent is able to find the optimal vector of action:

$$
y^{*}(r)=\left(y_{1}^{*}(r), y_{2}^{*}(r), \ldots, y_{n}^{*}(r)\right),
$$

where

$$
y_{i}^{*}(r)=r_{i} / \sum_{j \in N} r_{j}, i \in N
$$


Consider two options of agents information structure (hierarchy of beliefs) about the vector of their types: in the first case the agent $i \in N$ has the beliefs $r_{i j}>0$ about the types of other agents $j \neq i$; in the second case he has beliefs $r_{i j k}>0$ about these beliefs, $i, j, k \in N$.

Assume that each agent knows his own type and under the axiom of self-information (Novikov and Chkhartishvili, 2003) $\quad r_{i i}=r_{i}, r_{i i j}=r_{i j}, r_{i j j}=r_{i j}$, $i, j \in N$.

Under the existing beliefs each agent is able to predict what actions his opponents will choose, what will be their individual cost and total cost. If the actions are chosen iteratively, and the reality, observed by some agent, differs from his expectations, then he has to correct his beliefs adaptively.

The set of the parameters, observed by the $i$-th agent is referred to as his subjective game history and is denoted by $h_{i}, i \in N$. Subjective game history of the $i$-th agent may include observations of:

1) actions of other agents (assume that each agent always observes his own actions) $y_{-i}=\left(y_{1}, y_{2}, \ldots, y_{i-1}\right.$, $\left.y_{i+1}, \ldots, y_{n}\right)$;

2) individual cost of other agents $c_{-i}=\left(c_{1}, c_{2}, \ldots\right.$, $\left.c_{i-1}, c_{i+1}, \ldots, c_{n}\right)$;

3) total cost $-c=\sum_{i \in N} c_{i}$;

4) actions and individual cost of other agents $\left(y_{-i} ; c_{-i}\right)$;

5) actions of other agents and total cost $\left(y_{-i} ; c\right)$.

Two types of information structures $\left(r_{i j}\right.$ and $\left.r_{i j k}\right)$ and five options of the subjective game history (which are assumed the same for all agents) generate ten models, conventionally denoted 1-10 (see table 1).

$\underline{\text { Table 1. Models of team building }}$

\begin{tabular}{ccc}
$\begin{array}{c}\text { Subjective } \\
\text { game's } \\
\text { history }\end{array}$ & \multicolumn{2}{c}{ Information structure } \\
\hline$y_{-i}$ & Model 1 & $\left\{r_{i j k}\right\}$ \\
$c_{-i}$ & Model 2 & Model 6 \\
$c$ & Model 3 & Model 7 \\
$\left(y_{-i} ; c_{-i}\right)$ & Model 4 & Model 9 \\
$\left(y_{-i} ; c\right)$ & Model 5 & Model 10 \\
\hline
\end{tabular}

Consider procedures of the agents decision making. Under the information structure $\left\{r_{i j}\right\}$ the $i$-th agent may choose his action either following the procedure (1):

$$
y_{i}^{*}\left(\left\{r_{i j}\right\}\right)=r_{i} / \sum_{j \in N} r_{i j}
$$

or he can, estimating actions of his opponents by (2), find his action, leading to the required sum of actions:

$$
y_{i}^{*}\left(\left\{r_{i j}\right\}\right)=1-\sum_{j \neq i}\left(r_{i j} / \sum_{j \in N} r_{i j}\right), i \in N
$$

It is easy to verify, that procedures (2) and (3) are equivalent.

Under the information structure $\left\{r_{i j k}\right\}$ the $i$-th agent may, estimating actions of his opponents by (1)

$$
y_{i j}^{*}\left(\left\{r_{i j k}\right\}\right)=r_{i j} / \sum_{k \in N} r_{i j k}, j \in N
$$

find his action, leading to the required sum of actions:

$$
y_{i}^{*}\left(\left\{r_{i j k}\right\}\right)=1-\sum_{j \neq i}\left(r_{i j} / \sum_{k \in N} r_{i j k}\right), i \in N
$$

After describing the models of the agents' decision making in static, consider the dynamics of their collective behavior.

\section{DYNAMICS OF COLLECTIVE BEHAVIOR}

Assume that at each step (time period) each agent makes his decision, based on the information only about the previous step. Denote $W_{i}^{t}\left(h_{i}^{t}\right)-$ the current goal of the $i$-th agent in the period $t$, i.e. his beliefs $I_{i}^{t}$ about the types of the opponents, which can lead to the observed game history, $t=0,1,2, \ldots$, $i \in N$.

Let the agents initially have some beliefs $I_{i}^{0}$, and beliefs are modified in accordance with the following procedure:

$$
\begin{gathered}
I_{i}^{t+1}=I_{i}^{t}+\gamma_{i}^{t}\left(W_{i}^{t}\left(h_{i}^{t}\right)-I_{i}^{t}\right), \\
t=1,2, \ldots, i \in N
\end{gathered}
$$

where $\gamma_{i}^{t}$ - is a vector, which components - numbers from $[0 ; 1]$ are interpreted as the "sizes of steps towards the current goal" and provide the convergence of (6). As the beliefs of each agent are described by the finite set of parameters $r_{i j}$ or $r_{i j k}$, $i, j, k \in N$, then (6) defines "vector" procedure of the independent changes of the information structure components.

Now one has everything that is necessary to define the team correctly. Let's define a team as a set of agents, whose choices are in concordance with the hierarchy of their beliefs about each other. 


\section{RESULTS OF MODELS EXPLORATION}

Model 1. Assume that the agent $i$ has the information structure $\left\{r_{i j}\right\}$ and observes actions $x_{-i}$ of other agents. Denote the set of types of the i-th agent's opponents, which lead to the actions, chosen in accordance with (2), equal to the observed actions $x_{-i}$ :

$$
\begin{gathered}
\Omega_{i}^{1}=\left\{r_{i j}>0, j \in N \backslash\{i\} \mid r_{i j} / \sum_{j \in N} r_{i j}=x_{j},\right. \\
j \in N \backslash\{i\}\}
\end{gathered}
$$

Denote $w_{i j}^{t}\left(x_{-i}^{t}\right)-$ the $j$-th projection of the nearest to $\left(r_{i j}^{t}\right)_{j \in N \backslash\{i\}}$ point of the set $\Omega_{i}^{1}$. Then the dynamics of the $i$-th agent beliefs is described by

$$
\begin{gathered}
r_{i j}^{t+1}=r_{i j}^{t}+\gamma_{i j}^{t}\left(w_{i j}^{t}\left(x_{-i}^{t}\right)-r_{i j}^{t}\right), j \in N \backslash\{i\}, \\
t=1,2, \ldots, i \in N
\end{gathered}
$$

and his choice of actions will follow the procedure (2).

Model 2. Assume that the agent $i$ has the information structure $\left\{r_{i j}\right\}$ and observes the cost $c_{-i}$ of other agents.

Denote the set of types of the $i$-th agent's opponents, which lead under the actions chosen in accordance with (2), to the observed cost $c_{-i}$ :

$$
\begin{gathered}
\Omega_{i}^{2}=\left\{r_{i j}>0, j \in N \backslash\{i\} \mid c_{j}\left(r_{i j} / \sum_{j \in N} r_{i j}, r_{i j}\right)=c_{j},\right. \\
j \in N \backslash\{i\}\}
\end{gathered}
$$

Denote $w_{i j}^{t}\left(c_{-i}\right)-$ the $j$-th projection of the nearest to $\left(r_{i j}^{t}\right)_{j \in N \backslash\{i\}}$ point of the set $\Omega_{i}^{2}$. Then the dynamics of the $i$-th agent beliefs is described by (8), and his choice of actions will follow the procedure (2).

Model 3. Assume that the agent $i$ has the information structure $\left\{r_{i j}\right\}$ and observes the total cost $c$ of the agents.

Denote the set of types of the i-th agent's opponents, which lead to the observed total $\operatorname{cost} c$ :

$$
\begin{gathered}
\Omega_{i}^{3}=\left\{r_{i j}>0, j \in M\{i\} \mid c_{i}\left(y_{i}, r_{i}\right)+\right. \\
\left.+\sum_{j \in N \backslash\{i\}}\left[c_{j}\left(r_{i j} / \sum_{j \in N} r_{i j}, r_{i j}\right)\right]=c\right\}
\end{gathered}
$$

Denote $w_{i j}^{t}(c)$ - the $j$-th projection of the nearest to $\left(r_{i j}^{t}\right)_{j \in N \backslash\{i\}}$ point of the set $\Omega_{i}^{3}$. Then the dynamics of the i-th agent beliefs is described by (8), and his choice of actions will follow the procedure (2).
Models 4 and 5 are described analogously to models 1 and 2 .

Model 6. Assume that the agent $i$ has the information structure $\left\{r_{i j k}\right\}$ and observes actions $x_{-i}$ of other agents. Denote the set of types of the $i$-th agent's opponents, which lead to the actions, chosen in accordance with (4), equal to the observed actions $x_{-i}$ :

$$
\begin{array}{r}
\Omega_{i}^{6}=\left\{r_{i j k}>0, j \in N \backslash\{i\}, k \in N \mid\right. \\
\left.r_{i j} / \sum_{k \in N} r_{i j k}=x_{j}, j \in N \backslash\{i\}\right\}
\end{array}
$$

Denote $w_{i j k}^{t}\left(x_{-i}^{t}\right)$ - the $j k$-th projection of the nearest to $\left(r_{i j k}^{t}\right)_{j \in N \backslash\{i\}}$ point of the set $\Omega_{i}^{6}$. Then the dynamics of the $i$-th agent beliefs is described by

$$
\begin{gathered}
r_{i j k}^{t+1}=r_{i j k}^{t}+\gamma_{i j}^{t}\left(w_{i j k}^{t}\left(x_{-i}^{t}\right)-r_{i j k}^{t}\right), \\
j \in N \backslash\{i\}, t=1,2, \ldots, i \in N
\end{gathered}
$$

and his choice of actions will follow the procedure (5), i.e.:

$$
y_{i}^{t^{*}}\left(\left\{r_{i j k}^{t}\right\}\right)=1-\sum_{j \neq i}\left(r_{i j}^{t} / \sum_{k \in N} r_{i j k}^{t}\right), i \in N
$$

Model 6 is analogous to model 1 , model 7 - to model 2 and so on, hence the detailed description of models $7-10$ is omitted.

Thus, from each agents' point of view in model 1 there are $n-1$ equations with $n-1$ variables, in model 2: $n-1$ equations with $n-1$ variables, in model 3: one equation with $n-1$ variables, in model 4: $2(n-1)$ equations with $n-1$ variables, in model 5: $n$ equations with $n-1$ variables, in model 6: $n-1$ equations with $n(n-1)$ variables, etc.

\section{THE EXAMPLE}

Consider model 1 - the simplest among the introduced ten models - for three agents with separable quadratic cost functions: $c_{i}\left(y_{i}, r_{i}\right)=\left(y_{i}\right)^{2} / 2 r_{i}$. Then (7) leads to:

$$
\begin{aligned}
& w_{13}\left(x_{2}, x_{3}\right)=x_{3} r_{1} /\left(1-x_{2}-x_{3}\right), \\
& w_{12}\left(x_{2}, x_{3}\right)=x_{2} r_{1} /\left(1-x_{2}-x_{3}\right), \\
& w_{21}\left(x_{1}, x_{3}\right)=x_{1} r_{2} /\left(1-x_{1}-x_{3}\right), \\
& w_{23}\left(x_{1}, x_{3}\right)=x_{3} r_{2} /\left(1-x_{1}-x_{3}\right), \\
& w_{31}\left(x_{1}, x_{2}\right)=x_{1} r_{3} /\left(1-x_{1}-x_{2}\right), \\
& w_{32}\left(x_{1}, x_{2}\right)=x_{2} r_{3} /\left(1-x_{1}-x_{2}\right),
\end{aligned}
$$

Let $r_{1}=1,8 ; r_{2}=2 ; r_{3}=2,2$, and initial beliefs of the agents are the same and equals to 2,0. Optimal 
(minimizing objective total cost) is the following vector of actions: $(0,30 ; 0,33 ; 0,37)$.

Let the agents calculate by (2) actions of opponents, which minimize "subjective" total cost, then they compare observed and expected actions and change their beliefs proportionally to this difference with the coefficient of proportionality $\gamma_{i j}^{t}=0,25, i, j \in N$, $t=1,2, \ldots$

This procedure converges to the vector of actions $(0,316 ; 0,339,0,345)$ under the following beliefs of the agents: $\quad r_{12}=1,93<r_{2}, \quad r_{13}=1,94<r_{3}$, $r_{21}=1,86>r_{1}, \quad r_{23}=2,01<r_{3}, \quad r_{31}=2,02>r_{1}$, $r_{32}=2,17>r_{2}$. In spite of the incorrect beliefs, the situation is stable - expected actions coincide with observed actions.

Let under $r_{1}=1,8 ; \quad r_{2}=2 ; \quad r_{3}=2,2$ initial beliefs changed to

$$
\begin{gathered}
r_{12}^{0}=2, r_{13}^{0}=2,5, r_{21}^{0}=1,5, r_{23}^{0}=2,5, r_{31}^{0}=1,5, \\
r_{32}^{0}=2 .
\end{gathered}
$$

Then the same vector of actions: $(0,30 ; 0,33 ; 0,37)$ is objectively optimal. But the procedure converges to the vector of actions $(0,298 ; 0,3484,0,3524)$ under the following beliefs of the agents: $r_{12}=2,1>r_{2}$, $r_{13}=2,12<r_{3}, \quad r_{21}=1,71<r_{1}, \quad r_{23}=2,01<r_{3}$, $r_{31}=1,85>r_{1}, r_{32}=2,16>r_{2}$. Again, in spite of the incorrect beliefs, the situation is stable - expected actions coincide with observed actions.

Procedure (8) under the same initial point gives the following vector of actions: $(0,318 ; 0,341,0,341)$ under the following beliefs of the agents: $r_{12}=1,93<r_{2}, \quad r_{13}=1,93<r_{3}, \quad r_{21}=1,87>r_{1}$, $r_{23}=2,00<r_{3}, r_{31}=1,05>r_{1}, r_{32}=2,2>r_{2}$. Again, in spite of the incorrect beliefs, the situation is stable - expected actions coincide with observed actions.

The effect of the informational equilibrium stability (Novikov and Chkhartishvili, 2004) under incorrect beliefs has transparent explanation: the solution of the system of equations (7) is not unique. Let us define such situation as a false equilibrium. In fact, for example, in the case of two agents the system of three equations

$$
\left\{\begin{array}{l}
\frac{r_{12}}{r_{1}+r_{12}}=x_{2} \\
x_{1}+x_{2}=1 \\
\frac{r_{21}}{r_{2}+r_{21}}=x_{1}
\end{array}\right.
$$

with four variables $-r_{12}, r_{21}, x_{1}, x_{2}-$ has infinite number of solutions: $r_{12}=r_{1}\left(1 / x_{1}-1\right)$, $r_{21}=r_{2} x_{1} /\left(1-x_{1}\right), x_{2}=1-x_{1}, x_{1} \in(0 ; 1)$.

Note, that the transfer from model 1 to model 4 adds the information about the cost of the opponents and may narrows the set of the solutions. In the considered example unique simultaneous observation of agent's action and his cost allows to find agent's type unambiguously.

Consider the following example. Let there are two agents with types $r_{1}=1,5$ and $r_{2}=2,5$. Initial beliefs $r_{12}^{0}=1,8, r_{21}^{0}=2,2$ are incorrect. Final beliefs are $r_{12}=1,747 ; r_{21}=2,147$.

In this case subjective equilibria is $x_{1}=0,4614$; $x_{2}=0,5376$. The observed actions form informational equilibria as they correspond to beliefs of the agents (satisfy the system of equations (14)).

The set of subjective equilibrium for the example in hand is presented in figure 1 . The bold point marks the initial beliefs, the rhomb marks correct beliefs, the arrow points the change in agents beliefs about each other.

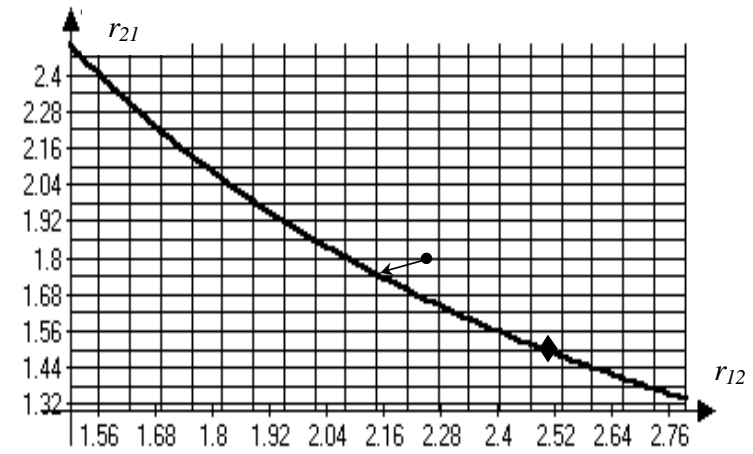

Fig. 1. The set of subjective equilibria

The system of equations (14) demonstrates, that all informational equilibrium, satisfying:

$$
r_{12} r_{21}=r_{1} r_{2}
$$

are stable. The set of beliefs $\left(r_{12} ; r_{21}\right)$, satisfying (15), is a hyperbola. An example of this hyperbola is presented in figure 2 for the case $r_{1}=2 ; r_{2}=1$.

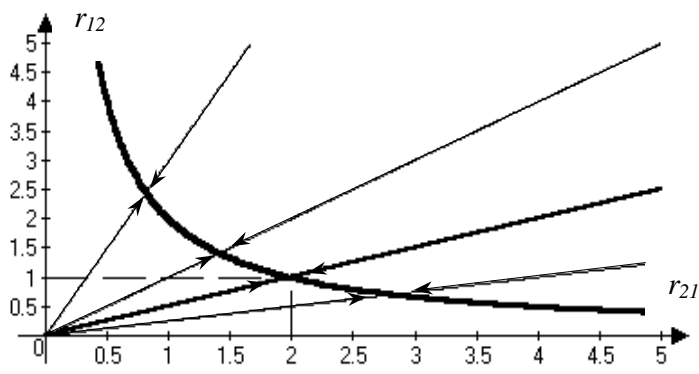

Fig. 2. Attraction sets of the subjective equilibria

One can not only find the set (15) of false equilibria, but to explore the set of their attraction: it follows from (8) that the dynamics of beliefs satisfies:

$$
\frac{\Delta r_{12}^{t}}{\Delta r_{21}^{t}}=\frac{\gamma_{12}^{t}}{\gamma_{21}^{t}} \frac{r_{12}^{t-1}}{r_{21}^{t-1}}, t=1,2, \ldots
$$


hence, under constant "steps" $\gamma$ trajectories will form lines, passing through zero. The angle of these lines is determined by the initial point (for example, any initial point, belonging to the bold line $r_{12}=r_{21} / 2$ in figure 2 , leads to the true equilibrium).

This fact seems interesting from the informational management (Novikov and Chkhartishvili, 2003, 2004) point of view - given the terminal point, one can find the set of initial points, leading to this final point.

\section{FALSE EQUILIBRIA}

Examples, considered above, bring up the following question: is the situation of false equilibrium typical? Let's generally answer this question for the model 1 .

Let the vector $r=\left(r_{1}, r_{2}, \ldots, r_{n}\right)$ of the agents types is common knowledge and corresponding optimal vector of actions $y^{*}(r)=\left(y_{1}^{*}(r), y_{2}^{*}(r), \ldots\right.$, $\left.y_{n}^{*}(r)\right)$ is unique. Thus, $n$ functions $\varphi_{i}: r \rightarrow y_{i}^{*}(r)$, $i \in N$, mapping vectors of types to the actions, are given. Now assume, that the described situation holds subjectively: each agent believes that some vector of types is common knowledge. Then the informational structure of the game is described by $N$ vectors like $\left(r_{i l}, r_{i 2}, \ldots, r_{i n}\right), i \in N$. Informational equilibrium $y^{*}=\left(y_{1}^{*}, y_{2}^{*}, \ldots, y_{n}^{*}\right)$ will be stable if each agent will observe the same actions, as he expected to observe. It means, that the following conditions are hold:

$$
\varphi_{i}\left(r_{j l}, r_{j 2}, \ldots, r_{j n}\right)=y_{i}^{*}(r), i, j \in N
$$

If the equilibrium $y^{*}$ is arbitrary, then (17) impose $n(n-1)$ restrictions on the informational structure. Moreover, if the true type of each agent is fixed, then one can find $n(n-1)$ variables $r_{i j}, i, j \in N, i \neq j$.

System of equations (17) is satisfied by the set of beliefs $r_{i j}$, such, that $r_{i j}=r_{j}$ for all $i$ and $j$. Thus, the problem of the false equilibrium existence under the given vector of agents true types $\left(r_{1}, r_{2}, \ldots, r_{n}\right)$ is reduced to the problem of the uniqueness of solution for the system of equations (17), which consists of $n(n-1)$ equations and the same number of unknown variables.

\section{CONCLUSION}

Consideration of the game-theoretical model of team building allows concluding that the stability of the team may be achieved both in the true stable informational equilibria and in the false ones. Transfer from false equilibria to the true equilibria requires additional information. Thus, methods of efficient team building are: learning through joint activity and providing maximal communication and all the essential information.

\section{REFERENCES}

Myerson, R.B. (1995). Game theory: analysis of conflict, Harvard Univ. Press, London.

Novikov, D.A. and Chkhartishvili A.G. (2003), Informational equilibrium: point belief structures. Automation and Remote Control, 10, 111122.

Novikov, D.A. and Chkhartishvili A.G. (2004), Models of reflexive decision making. Proceedings of XV International Conference on Systems Science, Wroclaw, Poland. 